\title{
Englisch soll die Landessprachen als Kongress- sprache lediglich ergänzen und nicht ersetzen
}

\author{
Thomas Hofera, \\ Monica Pongratz Guntli ${ }^{b}$ \\ a Dr. med., Dermatologie \\ und Venerologie FMH, Wettingen \\ b Leiterin Sekretariat SGDV
}

Korrespondenz:

Sekretariat SGDV

Postfach 782

CH-2002 Neuenburg

Tel. 0327214260

sgdv-ssdv@hin.ch

\section{Zusammenfassung}

In der medizinischen Fachliteratur dominiert Englisch. Englisch/Amerikanisch ist die offizielle Sprache internationaler Kongresse. In der Schweiz ertönt der Wunsch, dass man, der allgemein besseren Verständlichkeit wegen, Englisch als offizielle Sprache nationaler Kongresse einführt. Der schweizerischen demokratischen Gepflogenheit folgend, soll ein solcher Entscheid die Meinung der Mitglieder der SGDV berücksichtigen. Es erfolgt eine Umfrage. Die Beteiligung beträgt $84,2 \%$ ! Ein knappes absolutes Mehr von 53\%, bedingt durch das stimmliche Übergewicht der Deutschschweizer (die Tessiner Kolleginnen und Kollegen sprechen sich mit $77 \%$ dagegen aus!), akzeptiert die Aufnahme von Englisch als dritte offizielle Kongresssprache neben Deutsch und Französisch. Englisch als alleinige Kongress- sprache findet mit 9\% Ja-Stimmen-Anteil kaum Unterstützung. 92\% wünschen, dass die Diskussion neben englisch wie bis anhin in deutsch und französisch geführt wird. Neben der verbalen Kommunikation in der Muttersprache sind Textprojektionen in der jeweils anderen Landessprache oder aber in englisch erwünscht.

\section{Einleitung}

Im Zusammenhang mit der wirtschaftlichen wie auch kulturellen Globalisierung entwickelt sich Amerikanisch zur dominierenden Kommunikationshilfe. Einige Kantone der Schweiz, angeführt durch den bevölkerungsreichsten und wirtschaftlich dominanten Kanton Zürich, versuchen dieser Entwicklung zu begegnen, indem das Erlernen von Englisch demjenigen einer zweiten Landessprache der Schweiz, wie es bis

\section{Résultat d'un sondage de la Société suisse de dermatologie et de vénéréologie SSDV \\ Dans les congrès, I'anglais peut être utile, mais ne doit pas remplacer les langues nationales}

Dans la littérature médicale spécialisée, c'est l'anglais qui domine. L'anglais ou I'«américain» est la langue officielle des congrès internationaux. En Suisse, certains souhaiteraient, pour améliorer la communication, que la langue de Shakespeare devienne également l'une des langues officielles des congrès à l'échelle nationale. Conformément aux coutumes démocratiques de notre pays, il est nécessaire, avant de prendre une telle décision, de demander I'avis des membres de la SSDV. Un sondage a donc été mené, auquel $84,2 \%$ des membres ont participé! La proposition d'instaurer I'anglais comme troisième langue officielle des congrès, en complément de l'allemand et du français, a obtenu de justesse la majorité absolue avec $53 \%$ des suffrages. Le vote des membres de Suisse alémanique, plus nombreux, a bien entendu pesé dans la balance (nos collègues tessinois se sont exprimés à $77 \%$ contre la motion!).

La proposition d'introduire l'anglais comme unique langue de congrès n'a eu guère de succès, ne récoltant que $\mathbf{9} \%$ de votes favorables. $\mathbf{9 2} \%$ des personnes qui ont donné leur avis souhaitent que l'on continue, comme on l'a fait jusqu'à maintenant, à utiliser l'allemand et le français dans les discussions avec I'anglais en complément. Nous souhaitons également que, parallèlement aux communications en langue maternelle, les projections de textes à l'écran se fassent dans une autre des langues nationales ou en anglais. 
anhin seit Jahrzehnten üblich ist, vorgezogen wird. Dieses Vorgehen bricht abrupt mit einer Tradition und ist nicht unbestritten, wird aber von der Schweizerischen Konferenz der kantonalen Erziehungsdirektoren unterstützt [1], obwohl sie in einem für die Betroffenen, die Schüler, enorm wichtigen Fach, der Kommunikation, dem Ziel einer Harmonisierung der obligatorischen Schule wohl nicht entsprechen dürfte. In einem ersten Anlauf hat der Nationalrat am 21. Juni 2007 denn auch mit 112 zu 56 Stimmen versucht, im neu zu schaffenden Sprachengesetz des Bundes über die Reihenfolge der Fremdsprachen in der Schule dieser Entwicklung gesetzgeberisch Einhalt zu bieten.

In der medizinischen Fachliteratur dominiert Englisch. Englisch/Amerikanisch ist die offizielle Sprache internationaler Kongresse. Einzelne schweizerische medizinische Fachgesellschaften beginnen ihre nationalen Kongresse - entgegen der bis anhin üblichen Gepflogenheit, dass jeder Teilnehmer seinen Beitrag oder sein Diskussionsvotum in seiner Muttersprache mitteilen darf in der alleinigen offiziellen Kongresssprache Englisch/Amerikanisch durchzuführen. Obwohl die im Juni 2006 revidierte Weiterbildungsordnung (WBO) der FMH in Artikel 25 festhält, dass der schriftliche Teil der Facharztprüfung neben deutsch und französisch auf Gesuch hin auch auf italienisch abgelegt werden kann, begegnen einzelne Fachgesellschaften gerade diesem Wunsch mit einer Flucht nach vorn, indem die Facharztprüfung in Zukunft gar nicht mehr in einer Landessprache, sondern nur noch in englisch absolviert werden kann. Es wird somit vorausgesetzt, dass ein angehender schweizerischer Facharzt Englisch in Wort und Schrift beherrscht.

Vor diesem Hintergrund ist es verständlich, dass die offiziellen Kongresssprachen Deutsch und Französisch der SGDV zur Diskussion gestellt werden. Es ertönt der Wunsch, dass man, der allgemein besseren Verständlichkeit wegen, Englisch als offizielle Kongresssprache einführt. Um aber nicht voreilig kulturelle Schätze preiszugeben, muss der Umgang mit der Dynamik der Globalisierung, mit der Amerikanisierung auf sprachlicher Ebene, gelernt werden. Der schweizerischen demokratischen Gepflogenheit folgend, soll ein solcher Entscheid daher die Meinung der Mitglieder der SGDV berücksichtigen. Es erfolgt eine Umfrage.

\section{Methode}

Anfang Mai wurde den aktiven Mitgliedern der SGDV ein anonym zu beantwortender Fragebogen mit folgendem Inhalt zugeschickt:
- 1. Frage: Welches ist Ihre Muttersprache $(\mathrm{D}, \mathrm{F}, \mathrm{I})$ ?

- 2. Frage: Sollen wie bis anhin Deutsch und Französisch alleinige Kongresssprachen bleiben?

- 3. Frage: Soll neben Deutsch und Französisch Englisch als 3. Kongresssprache akzeptiert sein?

- 4. Frage: Soll Englisch alleinige Kongresssprache sein?

- 5. Frage: Soll die Diskussion wie bis anhin, je nach Muttersprache, in allen Kongresssprachen (evtl. inkl. Englisch) geführt werden?

- 6. Frage: Soll im Falle, dass Englisch alleinige Kongresssprache ist, die Diskussion nur in englisch erfolgen?

- 7. Frage: Soll im Falle, dass Englisch alleinige Kongresssprache ist, die Diskussion nebst englisch wie bis anhin je nach Muttersprache (D, F, I) geführt werden?

Sowohl von den Fragen 2 bis 4 wie 6 und 7 durfte jeweils nur je eine mit «Ja» beantwortet werden. Insgesamt wurden 354 Fragebogen, 227 in deutscher, 127 in französischer Sprache, verschickt (davon 12 in französischer bzw. 4 in deutscher Sprache an Mitglieder in der italienischen Schweiz).

\section{Resultate}

298 der 354 verschickten Fragebogen wurden bis Einsendeschluss Ende Juni 2007 zurückgeschickt. Die Beteiligung an der Umfrage betrug somit 84,2\%. 39 von ihnen konnten nicht berücksichtigt werden, da ihr Ausfüllen nicht den Vorgaben entsprach (doppeltes «Ja»). Somit konnten 259 (73,2\%: D 76\%, F $63 \%$, I 81\%) der verschickten Fragebogen verwertet werden.

- 99, d.h., 38\% (D 39\%, F 33\%, I 62\%) beantworteten die 2. Frage mit «Ja»;

- 138, d.h., 53\% (D 57\%, F 50\%, I 23\%) beantworteten die 3. Frage mit «Ja»;

- 23, d.h., 9\% (D 6\%, F 5\%, I 15\%) beantworteten die 4. Frage mit «Ja»;

- 237, d.h., 92\% (D 94\%, F 86\%, I 92\%) beantworteten die 5 . Frage mit «Ja»;

- 51, d.h., 20\% (D 17\%, F 26\%, I 23\%) beantworteten die 6 . Frage mit «Ja»;

- 192, d.h., 74\% (D 77\%, F 68\%, I 77\%) beantworteten die 7 . Frage mit «Ja».

\section{Diskussion}

Die Schweiz, im Zentrum Europas gelegen, stellt eine wichtige sprachliche Schweissnaht dar in der europäischen Nord-Süd- und Ost-West- 
Achse. Seit Jahrhunderten pflegt sie ihre Sprachenvielfalt. Neben dem Beherrschen der eigenen Muttersprache gehört das Erlernen von Grundkenntnissen in einer weiteren ihrer drei grossen Landessprachen zur Pflicht eines jeden Schülers, mit dem Ziel, die Kommunikation, das gegenseitige Verstehen zu pflegen. Gerade wurde im Kanton Graubünden mit der Annahme eines speziellen Gesetzes [2] durch den Souverän das Weiterbestehen der vierten Landessprache, des Rätoromanischen, im Sinne eines wichtigen Minoritätenschutzes bestätigt. Parallel zu diesen Bemühungen auf verbal-kommunikativer Ebene verbinden Strassen, alpenquerende Pässe und Tunnels die verschiedenen Sprachregionen. Die Pflege dieser Sprachenvielfalt wie auch die enormen finanziellen Aufwendungen für das Überwinden geographisch-topographischer Hindernisse fördern neben der sprachlichen und räumlichen Kommunikation das Selbstbewusstsein jeder Sprachregion und dadurch wiederum die Bereitschaft der Bewohner wie auch der Regionen $\mathrm{zu}$ gegenseitiger Wertschätzung und Akzeptanz. Vor diesem Hintergrund präsentiert sich die Schweiz sowohl touristisch, politisch, wissenschaftlich wie auch wirtschaftlich dem Vergleich mit dem Ausland: Beim «Travel and Tourism Competitiveness Report», dem ersten Index für die Wettbewerbsfähigkeit der Länder weltweit [3], belegt die Schweiz 2007 den ersten Platz. Auch im «Global Competitiveness Report» des World Economic Forum, der in erster Linie ökonomische Aspekte berücksichtigt, führt die Schweiz 2006-2007 vor Finnland und Schweden die Rangliste an [4].

In den letzten Jahren in Deutschland durchgeführte Umfragen zeigen, dass grosse Mehrheiten der deutschen Ärzteschaft gegen eine Amerikanisierung ihrer Fachzeitschriften und nationalen Kongresse sind. 84,7\% sprechen sich gegen rein englischsprachige inländische Fachzeitschriften aus. Für nationale Tagungen fordern 98,3\% die Landessprache als Kongresssprache [5]. Auf internationalen Kongressen in Deutschland wird von 84,3\% der Ärzteschaft Englisch als weitere Kongresssprache neben Deutsch akzeptiert. Hingegen lediglich eine Minderheit von 5,6\% wünscht Englisch als alleinige Kongresssprache. Die Resultate sind übrigens nicht abhängig vom Alter der Befragten [6]. Entsprechende Umfragen mit öffentlicher Publikation ihrer Resultate sind unseres Wissens in der Schweiz noch nie erfolgt. Die SGDV hat in der hier vorgestellten Abstimmung unter ihren Mitgliedern klar Stellung bezogen für die Aufrechterhaltung der Sprachenvielfalt. Der üblichen Usanz folgend, dass demokratisch erhobene Re- sultate in der Schweiz nicht auf ihre statistische Relevanz hin untersucht werden, um davon abhängig über ihre Aussagekraft zu urteilen, wird auch hier auf den Beizug eines Statistikers verzichtet. Ein knappes absolutes Mehr von 53\%, bedingt durch das stimmliche Übergewicht der Deutschschweizer (3. Frage), wünscht die Aufnahme von Englisch als dritter offizieller Kongresssprache neben Deutsch und Französisch. $38 \%$ hätten gerne den Status quo beibehalten. Englisch als alleinige Kongresssprache findet mit 9\% Ja-Stimmen-Anteil kaum Unterstützung. 92\% wiederum wünschen, dass nach Annahme von Englisch als dritter offizieller Kongresssprache die Diskussion neben englisch wie bis anhin in deutsch und französisch geführt wird. Die Suisse romande und die Deutschschweiz sind sich in ihrer Beurteilung weitgehend einig. Die 13 Stimmen aus dem Tessin jedoch sprechen sich deutlich (77\%) gegen die Aufnahme von Englisch als offizieller Kongresssprache aus (3. Frage). Die Erklärung für dieses Votum dürfte darin liegen, dass die Italienischsprachigen ihre medizinische Facharztausbildung entweder in der Suisse romande oder der Deutschschweiz absolviert und somit bereits einen erheblichen Mehraufwand in bezug auf das Erlernen einer weiteren Landessprache betrieben haben als die Romands oder die Deutschschweizer. Zudem sind die Tessiner in den meisten Fällen sogar in drei Landessprachen gewandt zu Hause. Ein ähnliches Engagement wird von ihnen somit von den Nicht-Italienisch-Sprechenden erwartet, bevor man eine Nichtlandessprache wie Englisch als offizielle Kongresssprache einführt. Das Ja von 53\% zu Englisch als dritter offizieller Kongresssprache muss somit gekoppelt sein mit einer weitgehenden Berücksichtigung der Wünsche der italienischsprechenden Minorität: Englisch als Kongresssprache soll die Ausnahme bleiben (für nicht deutsch- oder französischsprachige, ausländische Referenten). Die Fragen und Voten der Diskussion erfolgen in freier Sprachwahl. Wünschenswert ist, dass neben der verbalen Kommunikation in der Muttersprache die Textprojektionen in der jeweils anderen Landessprache oder aber in englisch präsentiert werden. Dies dürfte als willkommener Nebeneffekt sogar beitragen zu einer qualitativen Verbesserung der durchschnittlichen mündlichen Vortragsart, die heute zu häufig lediglich der schriftlichen Vorgabe folgt.

Das hier vorgestellte Umfrageresultat spiegelt die Wertschätzung der gegenseitigen sprachlichen Kultur wider und verschliesst sich gleichzeitig nicht gegenüber einer vernünftigen kommunikativen Erweiterung dort, wo es sinnvoll 
ist. Für leitende Gremien medizinischer Fachgesellschaften, aber auch Mitglieder nationaler Gremien, die über die Weiter- und Fortbildung ihrer Ärzteschaft befinden, dürften die hier vorgestellten Resultate von Interesse sein. Medizin hat sehr viel mit Sprache zu tun. Die Sprache spielt eine zentrale Rolle in der Kommunikation zwischen Patient und Arzt. Medizin als Wissenschaft und Kunst hat aber primär nichts mit Kenntnissen in einer Fremdsprache zu tun. Diese Feststellung findet ihre Bestätigung darin, dass lediglich 20\% der deutschen Ärzteschaft, unabhängig von ihrem Alter, in einer Selbstdeklaration angeben, über sichere englische Sprachkenntnisse in Wort und Schrift zu verfügen $[5,6]$. Daten, die über die Englischkenntnisse schweizerischer praktizierender oder angehender Fachärzte informieren würden, gibt es keine.

«Sprache ist Kultur und das wichtigste Mittel ethnischer Identität. Sie ermöglicht als Instrument des Denkens und der Erkenntnis den Erwerb, die Konservierung und die Vermittlung von Wissen. Sie ist das wichtigste Mittel der Kommunikation, über das der Mensch verfügt» [7].
Die Schweiz hat ihre ausgezeichnete Positionierung im Wettbewerb mit allen Ländern der Welt ohne Frühenglisch und Amerikanisierung erreicht. Dazu wesentlich beigetragen hat ihre Kreativität, die ihrer ausgezeichneten Sprachenkultur entspringt.

\section{Literatur}

1 Pressedienst des Generalsekretariats EDK. Harmonisierung der obligatorischen Schule: HarmoS-Konkordat einstimmig verabschiedet. Pressemitteilung vom 15. Juni 2007.

2 Kantonale Volksabstimmung vom 17. Juni 2007 zum Sprachengesetz des Kantons Graubünden.

3 Blank J, Chiesa T. World Economic Forum. Travel and Tourism Competitiveness Report 2007: Executive Summary.

4 Global Competitiveness Report 2006-2007. World Economic Forum.

5 Haße W, Fischer RJ. Ärzteschaft gegen Anglisierung in der Medizin. Ergebnisse einer Umfrage. Dtsch Med Wochenschr. 2003;128:1338-41.

6 Haße W. Sprachwechsel in der Medizin. Ärzteblatt Sachsen. 2004;(2):55-7.

7 Blanke D. Sprachimperialismus oder alternative Sprachenpolitik in Europa? Vortrag. AttacSommerakademie 2004. Dresden, 30.7.-5.8.2004. 